\title{
La regulación emocional en los trastornos emocionales, una piedra nodal para los abordajes transdiagnósticos: una revisión de la literatura
}

\author{
JAVIER FERNÁNDEZ-ALVAREZ \\ fernanja@uji.es \\ AMANDA DÍAZ-GARCÍA \\ amdiaz@uji.es \\ AlBERTo GonZÁLEZ-RoBles \\ vrobles@uji.es \\ CRISTINA BOTELLA \\ botella@uji.es \\ AZUCENA GaRcía-Palacios \\ azucena@uji.es
}

\section{Resumen}

Introducción: La regulación emocional se ha convertido en un tema de creciente interés, tanto a nivel de investigación básica (Tamir, 2011) como en diversos ámbitos aplicados, fundamentalmente el clínico (Aldao, Nolan-Hoeksema y Schweizer, 2010). Si bien la regulación emocional ha sido estudiada en un amplio conjunto de entidades psicopatológicas, una importante proporción de los desarrollos están vinculados con los trastornos emocionales. En ese sentido, la producción exponencial en relación a la regulación emocional en depresión y ansiedad requiere de notables esfuerzos teóricos para integrar los resultados empíricos. Este trabajo se propone presentar una integración de los principales aportes de la regulación emocional en el campo de los trastornos emocionales, enfatizando su importancia para los abordajes transdiagnósticos. Con dicho objetivo se ha realizado una revisión de la literatura a través de una búsqueda ascendente (ancestry approach). Método: Se utiliza el modelo de regulación emocional propuesto por Gross (1998) para organizar la siguiente revisión, con las innovaciones que aporta la revisión extendida de dicho modelo (Sheppes, Suri y Gross, 2015). Resultados: A través del análisis de la bibliografía se destaca la predominancia del estudio de estrategias de regulación emocional individuales y encubiertas tales como la supresión, la reevaluación, la aceptación o la rumiación. Se plantean los desafíos futuros en el campo de estudio de la regulación emocional en la psicopatología.

Palabras clave: regulación emocional, transdiagnóstico, trastornos emocionales 


\section{Abstract}

Introducction: Emotion regulation has become a topic of increasing interest, both at the level of basic research (Tamir, 2011) and applied fields, mainly clinical (Aldao, Nolan-Hoeksema y Schweizer, 2010). While emotional regulation has been studied in a wide range of psychopathological entities, a significant proportion of the developments are linked to emotional disorders. In that sense, the exponential production in relation efforts to integrate the existing empirical findings. This work aims to present an integration of the main contributions of emotion regulation in the field of emotional disorders, emphasizing its importance for transdiagnostic approaches. With this objective a review of the literature through an ancestry approach was carried out. Method: The emotion regulation model proposed by Gross (1998) is taken to organize the next revision, linking the content to some of the innovations provided by the extended model (Sheppes, Suri y Gross, 2015). Results: In the light of the findings a prevalence of individual and covert strategies of emotion regulation such as suppression, reappraisal, acceptance or rumination stands out. Future prospects of the field of emotion regulation are considered.

Keywords: emotion regulation, transdiagnostic approach, emotional disorders

\section{Introducción}

La regulación emocional se ha convertido en un tema de creciente interés, tanto a nivel de investigación básica (Tamir, 2011) como en diversos ámbitos aplicados, fundamentalmente el clínico (Aldao, Nolan-Hoeksema y Schweizer, 2010). Si bien la regulación emocional ha sido estudiada en un amplio conjunto de entidades psicopatológicas, una importante proporción de los desarrollos están vinculados con los trastornos emocionales. En ese sentido, la producción exponencial en relación a la regulación emocional en depresión y ansiedad requiere de notables esfuerzos teóricos para integrar los resultados empíricos. Este trabajo se propone presentar una integración de los principales aportes de la regulación emocional en el campo de los trastornos emocionales, enfatizando su importancia para los abordajes transdiagnósticos. Con dicho objetivo se ha realizado una revisión de la literatura a través una búsqueda ascendente (ancestry approach). Se utiliza el modelo de regulación emocional propuesto por Gross (1998) para organizar la siguiente revisión, con las innovaciones que aporta la revisión extendida de dicho modelo (Sheppes y cols., 2015). A través del análisis de la bibliografía se destaca la predominancia del estudio de estrategias de regulación emocional individuales y encubiertas tales como la supresión, la reevaluación, la aceptación o la rumiación. Se plantean los desafíos futuros en el campo de estudio de la regulación emocional en la psicopatología.

\section{Método}

Se ha realizado una revisión de la literatura a partir de una búsqueda computarizada a través de las siguientes bases de datos: Scopus, PubMed y Web of Science, para artículos publicados entre el 2005 y el 2016 utilizando la siguientes combinación de palabras clave: «emotion regulation AND emotional disorders». De los resultados se evaluaron las publicacio- 
nes más relevantes utilizando los siguientes criterios: factor de impacto de la revista o cantidad de citas del artículo y listado de referencias en los artículos evaluados, así como los libros de referencia sobre la temática. Es decir, se llevó a cabo el procedimiento de búsqueda ascendente (ancestry approach). Para organizar la presente revisión, se utilizó el modelo de regulación emocional propuesto por Gross (2014), con las innovaciones que aporta la revisión del modelo en su versión extendida (Sheppes y cols., 2015).

\section{Resultados}

La regulación emocional se define como las estrategias que los individuos realizan para influenciar qué emociones se experimentan, así como cuándo y cómo se las expresa (Gross, 1998). En los últimos años, se ha identificado la relación estrecha existente entre determinados modos de desplegar el proceso regulatorio de las emociones y diversas entidades psicopatológicas (Kring y Sloan, 2010). Particularmente, se ha comprobado que las estrategias de regulación emocional inadecuadas son importantes factores que contribuyen de forma significativa al desarrollo y mantenimiento de los síntomas de ansiedad y depresión (Aldao, NolanHoeksema y Schweizer, 2010).

El hallazgo de procesos transdiagnósticos constituye una indudable posibilidad de avanzar en el desarrollo de una alternativa conceptual en el campo psicopatológico, que atraviesa una franca disputa epistémica, conceptual y pragmática en la clasificación de las entidades nosológicas (Lilienfeld y Treadway, 2016). Los abordajes transdiagnósticos permiten incorporar la noción de que la naturaleza de los desórdenes mentales subyace en procesos comunes que exceden a la mera manifestación sintomática. De hecho, los manuales diagnósticos que continúan basando los principios taxonómicos en aspectos sintomatológicos han mostrado ser poco útiles, pues no logran resolver la enorme dificultad de clasificar desórdenes específicos que terminan engrosando los listados de trastornos no especificados. De este modo, una perspectiva transdiagnóstica permite generar un marco de referencia con una conceptualización flexible que facilita un modelo comprensivo de acuerdo a los complejos patrones de comorbilidad presentes en la psicopatología (Aldao, 2012).

Dada la tradición nosológica que heredó la psicopatología por su nacimiento naturalista y psiquiátrico, la comorbilidad es el aspecto que más ha resaltado la naturaleza común de los trastornos emocionales, lo cual, a su vez, ha estimulado el estudio de procesos transdiagnósticos subyacentes. Los estudios epidemiológicos muestran que un $60 \%$ de las personas que sufren un trastorno de ansiedad presentan un cuadro comórbido de ansiedad o de depresión. Cuando se analiza la comorbilidad en la trayectoria vital de las personas, la comorbilidad se eleva hasta el 76 \% (Brown, Campbell, Lehman, Grisham y Mancill, 2001). En este sentido, la regulación emocional se ha constituido como una aportación fundamental para el estudio de factores transdiagnósticos en los trastornos emocionales, en la línea de los planteamientos defendidos por Brown y Barlow (2009) acerca del papel común del afecto y de dimensiones de personalidad como el neuroticismo y la extraversión.

La regulación emocional se lleva a cabo siempre a través de distintas estrategias que se han ido clasificando a lo largo de los años en diversas categorías. Por ejemplo, según los momentos, se dividen en focalizadas en el momento antecedente (incluye la selección de la situación, la modificación de la situación, la focalización de la atención y el reencuadre cognitivo de una situación) o focalizadas en la respuesta (la modulación según el modelo clásico de Gross de 1998). Así como las estrategias adaptativas se las considera como tales por su naturaleza efectiva en el despliegue regulatorio, asociándose negativamente con medidas generales psicopatológicas, las estrategias antecedentes a la respuesta emocional parecen resul- 
tar métodos efectivos para la regulación en el corto plazo, mientras que las estrategias posteriores a la respuesta emocional tenderían a ser contraproducentes (Hofmann, 2014). Además del momento de inicio, una estrategia emocional puede variar en función del lugar en donde se sitúa (interno o externo), la motivación del resultado que se quiere conseguir (instrumental o hedónica), el nivel de conciencia (implícita o explícita), la direccionalidad de la regulación que puede ser a nivel de duración, intensidad y frecuencia, tanto para emociones positivas como negativas, y, por último, el apoyo que se busca para la resolución de una situación problemática (en uno mismo o en los otros).

De este modo, las estrategias a través de las que se despliega el proceso regulatorio de las emociones pueden derivar en consecuencias adaptativas o desadaptativas. Si bien se tiende a considerar que determinades estrategias son habitualmente adaptativas (como la reestructuración cognitiva o la aceptación) o desadaptativas (como la supresión o la rumiación), lo que realmente resulta explicativo de la aparición de estrategias desadaptativas es la flexibilidad que supone la capacidad de poner en contexto y evaluar consecuentemente qué tipo de categoría regulatoria, qué estrategia, en qué momento y con qué intensidad se debe llevar a cabo el proceso regulatorio (Bonanno, Papa, Lalande y cols., 2004; Aldao, Sheppes y Gross, 2015). Aldao y cols. (2015) identifican dos tipos de flexibilidad: aquella que interfiere con el objetivo y conlleva una consecuencia desadaptativa y otra que facilita el objetivo y, por lo tanto, deriva en una consecuencia adaptativa. Así mismo, el aspecto adicional consiste en poseer una cantidad óptima de estrategias regulatorias para la identificación, la selección y la implementación del proceso regulatorio emocional (Sheppes y cols., 2015). En definitiva, lo que queda claro es que la posibilidad de establecer patrones adaptativos de regulación emocional no puede establecerse a priori, sino que es necesaria una evaluación contextual en la que se produce dicha regulación. Las personas flexibles tenderán a adaptarse mejor a los diversos contextos, pudiendo desplegar las herramientas regulatorias en los momentos precisos (Aldao y Tull, 2015).

En cuanto a las estrategias más estudiadas en la literatura, cabe señalar las siguientes: la supresión, la reevaluación cognitiva, la aceptación, la rumiación y la evitación atencional (Aldao y Nolan-Hoeksema, 2010). La focalización en estas estrategias constituye una evidencia contundente de cómo se ha relegado el estudio de los posibles factores regulatorios externos y contextuales (Hofmann, 2014). De hecho, en la reconceptualización presentada recientemente por Sheppes y cols. (2015) se enfatizan tres aspectos fundamentales: a) el rol que juega la interacción respecto a la generación de la emoción en un proceso isomórfico, b) el despliegue temporal que implica toda regulación de la emoción y c) la complejización, a través de establecer tres momentos en los que se puede desplegar la regulación (identificación, selección e implementación). En cada una de las etapas pueden ocurrir déficits según cada uno de los tres elementos regulatorios que identifican Sheppes y cols., (2015): percepción, valuación y acción, elementos que forman parte de cada una de las etapas (identificación, selección e implementación).

La aportación fundamental para la psicopatología de la identificación y la selección consiste en permitir distinguir más sutilmente el foco donde la regulación emocional no se despliega de un modo adecuado. Mientras que la implementación apunta meramente a la ejecución, la identificación describe la percepción acerca de la situación y las estrategias disponibles. Por último, la selección hace referencia al proceso de escoger, precisamente, alguna de las estrategias identificadas en el menú. A su vez, en el nuevo modelo se mantienen las cinco tácticas identificadas en el modelo original, que dan lugar a las estrategias específicas que se han estudiado en relación a la psicopatología en general y a los trastornos emocionales en particular: la selección de la situación, la modificación de la situación, el despliegue atencional, el cambio cognitivo y la modulación de la respuesta (Sheppes y cols., 2015). 
Selección de la situación desadaptativa.Evitación: la evitación situacional es una de las pocas estrategias estudiadas en profundidad que son manifiestas (overt strategies). Investigaciones como la de Aldao, Jazaieri, Goldin y Gross (2014) han estudiado el papel desadaptativo que tiene este tipo de regulación emocional en los trastornos emocionales. La evitación situacional se vincula con la evitación experiencial, que ha sido el constructo más estudiado, sobre todo por su inclusión en las terapias de tercera generación (Hayes, 2004). Aunque puede ser discutible si la evitación experiencial incluye a la situacional, como afirman Boulanger, Hayes y Pistorello (2010), el hecho de no seleccionar una situación (es decir, evitarla) puede ser un proceso de down-regulation en situaciones que se perciben como altamente amenazantes (un grupo de gente, un lugar abierto, un lugar cerrado, una actividad, etc.). La evitación experiencial, en ese sentido, puede implicar cualquiera de las cinco etapas identificadas por Gross, Boulanger y cols. (2010): en las etapas de selección y modificación se podría producir una evitación experiencial aprendida a través de condicionamiento. El problema se presenta cuando este proceso se aprende de modo rígido y se generaliza a patrones de comportamiento alexitímicos, anhedónicos y disociativos. Del mismo modo, se puede presentar una evitación de la concentración, que respondería al tercer momento de la regulación emocional de Gross. La distracción excesiva está asociada con la rumiación y la preocupación excesiva (se abordan más abajo), que se relacionan íntimamente con los trastornos emocionales (Nolen-Hoeksema, Wisco y Lyubomirsky, 2008). Respecto a la evitación experiencial en el cuarto momento del proceso regulatorio, la reevaluación cognitiva puede estar mediada por la evitación experiencial. Por último, la evitación focalizada en la respuesta del proceso emocional incluye la supresión o alteración de respuestas conductuales a una emoción elicitada. Entre ellos se incluyen los intentos por controlar los informes verbales, las conductas afectivas y las respuestas fisiológicas.

Focalización desadaptativa de la atención. La rumiación es una estrategia de regulación emocional muy estudiada en cuanto a su relación con los trastornos emocionales (McLaughlin y Nolan-Hoeksema, 2011). Se la conceptualiza como un proceso de pensamiento que supone pensar de manera repetitiva y pasiva sobre los propios síntomas de malestar y sus posibles causas (Nolan-Hoeksema y cols., 2008). La rumiación suele estar presente en la depresión y la ansiedad generalizada y da lugar a un incremento del pensamiento negativo, perturba la resolución eficaz de los problemas, interfiere con la conducta instrumental y puede dar lugar a una erosión de los vínculos sociales (Aldao, 2012). Las mujeres utilizan más la estrategia de rumiación que los hombres, aspecto que pudiera estar vinculado con las tasas más elevadas de depresión y ansiedad en mujeres en comparación con los hombres (Johnson y Whisman, 2012). Por otro lado, la rumiación parece estar más ligada a la depresión que a la ansiedad, si bien tiene relación con ambos trastornos (Aldao y cols., 2010).

La rumiación no es intrínsecamente desadaptativa; ahora bien, se ha comprobado que en las personas con depresión la presencia de rumiación da lugar a un incremento de la interpretación negativa de los eventos pasados, lo cual incrementa los niveles de malestar general (Nolan-Hoeksema y cols., 2008). Las personas que utilizan dicha estrategia indican que lo hacen porque piensan que de este modo logran una mayor autoconciencia de su situación vital, de sus problemas y de supuestas posibles soluciones (Papageorgiou y Wells, 2001). Nolan-Hoeksema (2000) ha estudiado la relación entre la rumiación y los trastornos emocionales y ha comprobado que participantes sanos que puntuaban alto en rumiación tendían a desarrollar al año siguiente más sintomatología depresiva que los que no utilizaban la rumiación como mecanismo regulatorio. En esta misma línea, también se ha identificado la rumiación como un factor predisponente al inicio y mantenimiento de la depresión, siendo, por tanto, un objetivo específico en los tratamientos para esta condición clínica (Watkins, 2015). 
Cambio cognitivo desadaptativo. La reevaluación cognitiva consiste en la posibilidad de pensar acerca de una situación de modo diferente con el objetivo de modificar su impacto emocional. Habitualmente, está considerada como la estrategia más adaptativa cuando se aplica eficazmente y constituye la piedra nodal de la teoría cognitiva para explicar la aparición de muchos trastornos psicológicos, particularmente la depresión y la ansiedad (Beck y Haigh, 2014). En la mayoría de estudios, se identifica la reevaluación cognitiva como una estrategia adaptativa (véase, por ejemplo, Aldao, Nolan-Hoeksema y Schweizer, 2010). La posibilidad de reencuadrar la experiencia que elicita una emoción determinada o un estímulo (usualmente negativo) es una estrategia que resulta habitualmente adaptativa para alterar el impacto negativo que provoca. Según McRae, Ciesielsky y Gross (2012) existen ocho modos distintos en los que se puede reevaluar cognitivamente una situación. Al utilizar esta estrategia, la intensidad emocional puede ser mitigada (down-regulated) a través del pensamiento. La falta de flexibilidad cognitiva que caracteriza a los individuos con trastornos emocionales hace que la reevaluación sea poco utilizada como estrategia regulatoria y, por ello, se observa una correlación negativa con los trastornos emocionales. Nezlek y Kuppens (2008) han mostrado que la relación entre la reevaluación y una alta autoestima está mediada por el afecto positivo.

En cuanto a las emociones positivas, en la depresión las personas que sufren depresión tienen dificultades para imaginarse escenarios positivos futuros y, cuando logran visualizarlos, los valoran de manera menos positiva que los sujetos sanos. En un estudio realizado se encontró que, en comparación a los sujetos control, el grupo de personas con depresión mostraba dificultades para reevaluar estímulos positivos cuando era entrenado para generarlas de modo explícito en respuesta a imágenes placenteras (Carl y cols., 2013).

Modulación desadaptativa de la respuesta. La aceptación se ha conceptualizado como la habilidad para permanecer en contacto con los sentimientos, pensamientos y sensaciones físicas sin el objetivo de intentar modificarlas (Hayes y cols., 1999). Esta estrategia se debe asociar al proceso de modulación de la respuesta o monitoreo en el marco conceptual de Gross, lógicamente después de la etapa de implementación. Eifert y Heffner (2003) identifican la aceptación como una estrategia que permite reducir la ansiedad y la evitación conductual. Así mismo, en un estudio realizado por Campbell-Sills, Barlow, Brown y Hofmann (2006) se comparó la respuesta diferencial que generaba una estrategia de regulación de aceptación frente a la supresión de la situación displacentera y se observó que la aceptación daba lugar a un menor afecto negativo y una disminución de la respuesta cardíaca (arousal ansioso).

Se ha encontrado que la no aceptación está asociada a la ansiedad pero no a la depresión. Se considera la aceptación como un mecanismo opuesto a la supresión, que ha demostrado ser una estrategia desadaptativa. Por ejemplo, se ha comprobado que, en ocasiones, se genera un efecto paradójico cuando se intenta suprimir la emoción por su naturaleza displacentera, ya que lo que se logra es incrementar los niveles de malestar y arousal en comparación con los individuos que no utilizan tal estrategia (Abramowitz, Tolin y Street, 2001). También se ha comprobado que los pacientes con ansiedad que logran incorporar la aceptación como estrategia de regulación emocional reducen los niveles de malestar subjetivo y de evitación conductual. Esto resulta importante ya que sería un factor de vulnerabilidad atendiendo al esquema de la triple vulnerabilidad de Brown y Barlow (2009) en el que precisamente la evitación conductual, el afecto negativo y el neuroticismo son factores predisponentes para el desarrollo y mantenimiento de todos los trastornos emocionales. ${ }^{1}$ Existen otras respuestas desadaptativas que tienen que ver con estrategias manifiestas (overt strategies), como pueden ser el consumo de alcohol, los conflictos interpersonales, el comer o incluso ver la televisión de forma excesiva. Por ejemplo, en un estudio reciente en relación a estrategias mani-

1. Estos trabajos experimentales se pueden encontrar en Eifert y Heffner (2003) y Wolfgast (2012). 
fiestas y encubiertas se pone de manifiesto que las estrategias manifiestas (como el uso de sustancias, la búsqueda de ayuda, pelear con otros y evitar situaciones) están más relacionadas con la presencia de psicopatología que las estrategias encubiertas. En el caso de los trastornos de ansiedad, se ha observado una relación significativa con la presencia de estrategias como tomar alcohol y pelear con otros (Aldao y Dixon-Gordon, 2015).

La supresión es una estrategia focalizada en la respuesta que tiene como objetivo desprenderse de pensamientos, conductas y sentimientos de los que la persona es muy consciente. Si bien en ciertas ocasiones puede resultar efectiva su implementación (por ejemplo, la supresión expresiva en la down-regulation de expresiones faciales cuando se busca mantener o incrementar la intensidad de las experiencias subjetivas y el arousal fisiológico), en general es una estrategia que resulta desadaptativa. Por ejemplo, Campbell-Sills y cols. (2006) muestran cómo la supresión lleva a incrementar las tasas de ritmo cardíaco y el afecto negativo en comparación con un grupo que reevaluaba. La supresión puede estar dirigida a la inhibición conductual de la expresión emocional (expressive suppression) o a atenuar los sentimientos subjetivos que provoca una emoción (emotion suppression). Mientras que la expresión emocional puede ser efectiva para la supresión conductual, puede incrementar el arousal fisiológico y reducir la intensidad de la experiencia subjetiva de la emoción (Campbell-Sills, Ellard y Barlow, 2013). En determinadas circunstancias, la supresión ha mostrado ser efectiva, como por ejemplo después de situaciones traumáticas, ya que en ese momento la supresión permite reducir el malestar y las intrusiones posteriores. Si bien existen diferentes tipos de supresión, la supresión del pensamiento es la más relacionada con la presencia de psicopatología, particularmente los trastornos emocionales (Purdon, 1999). Se ha observado que la supresión emocional predice mejor resultado en psicoterapia que la reevaluación; sin embargo, la evidencia que aporta el estudio es contradictoria (Scherer, Boecker, Pawelzik, Gauggel y Forkmann, 2015). Esto apoya el énfasis dado por Aldao y Tull (2015) en relación al papel contextual y la flexibilidad como los dos aspectos más relevantes para determinar la adaptabilidad o no del despliegue de regulación emocional. Las personas que sufren un trastorno emocional, por lo general, experimentan emociones aversivas y agobiantes. Cabe pues hipotetizar que les resulta difícil la regulación una vez ya ha sido elicitada la emoción. Así mismo, se produce una disminución de la intensidad (down-regulation) de la emoción positiva en tanto hay una focalización en los pensamientos con contenido negativo o en las posibles consecuencias negativas. En este sentido, se identifica un incremento excesivo down-regulation de las emociones positivas (Carl y cols., 2013).

Particularmente, en la depresión se ha observado que los individuos tienen una notable dificultad para mantener respuestas emocionales positivas. Este aplanamiento de la capacidad de expresión emocional positiva parece ser un rasgo de muchos pacientes con depresión, por lo que se lo considera más un factor de vulnerabilidad que una consecuencia del trastorno. Por otro lado, el saboreo, una estrategia que implica la capacidad de disfrute de una situación (Bryant y Veroff, 2007), se ha visto disminuida en la depresión. Lo mismo se ha identificado con la rumiación positiva (Feldman y cols., 2008). En el caso de la ansiedad, se ha encontrado poca evidencia empírica, destacándose el arousal (como la activación del simpático) y la aprehensión (como el worry) como aspectos asociados con una menor capacidad de respuesta emocional positiva ante estímulos placenteros. Al igual que en la depresión, se observa incremento del down-regulation y una disminución de la up-regulation en las emociones positivas. 
Ahora bien, ¿cuáles son las limitaciones que presenta el campo de la regulación emocional? En primer lugar, cabe destacar que se ha prestado escasa atención a los aspectos interpersonales y contextuales que se vinculan con la regulación emocional ${ }^{2}$ (Hofmann y cols., 2014). En una reciente revisión, Gross (2015) enfatiza la necesidad de profundizar en el estudio de los aspectos contextuales. En consonancia con la concepción intraindividual de la regulación emocional, se ha hecho énfasis en las estrategias individuales y, sobre todo, las encubiertas. Si bien es necesario seguir ahondando en su estudio, Aldao y Dixon-Gordon (2013) insisten en que debe proliferar el estudio de las estrategias manifiestas como tomar alcohol, discutir con otros, evitar situaciones o buscar ayuda. Por ejemplo, estos autores comprobaron que discutir con otros era un factor asociado negativamente con la depresión, pero positivamente con el arousal ansioso; o que el consumo de alcohol como estrategia regulatoria manifiesta (overt) se asociaba positivamente con la ansiedad social.

La pregunta central que plantea Aldao (2012) es estudiar qué aspectos son transdiagnósticos de modo invariante y cuáles de modo variable. En otras palabras, ¿las estrategias de regulación emocional se dan de modo equivalente en todos los trastornos emocionales? Por ejemplo, el afecto positivo anteriormente descrito como un factor transdiagnóstico en los trastornos emocionales parecería no serlo de todos sino de algunos específicos, como la depresión mayor y la ansiedad social. Además, en los casos en los que la estrategia es la misma, también sería necesario identificar la variabilidad de su identificación y selección (creencias que tiene la persona respecto a su autoeficacia utilizando la estrategia), así como la implementación (la eficacia real llevándola a cabo). Por ejemplo, los individuos que padecen depresión y ansiedad social, en comparación con sujetos control sanos, utilizan la supresión expresiva y la rumiación de un modo excesivo y subutilizan la reevaluación. Ahora bien, si se compara el grupo de personas con diagnóstico de depresión y el grupo de pacientes con ansiedad social, se detecta que el primer grupo utiliza más frecuentemente la rumiación y menos la reevaluación que la ansiedad social. Por el contrario, este segundo grupo informa en mayor medida la utilización de supresión expresiva.

En este sentido, cobra particular interés el nuevo modelo presentado por Gross y cols., (2014), pues supone poner en marcha la ardua tarea de vincular las funciones básicas cognitivas alteradas en cada una de las etapas del proceso regulatorio, lo cual puede llevar a una mayor distinción entre trastornos específicos. Por ejemplo, en la identificación, los ataques de pánico se producen en el primero de los tres momentos, la percepción, precisamente por la sobrerrepresentación de los signos pequeños y sutiles de los estados emocionales que experimenta la persona. En cambio, en la implementación se identifica en la valuación: la preocupación excesiva (worry) que se observa en el trastorno de ansiedad generalizada y en el que se desarrolla una evaluación positiva de tácticas regulatorias desadaptativas.

Por último, cabe destacar el papel que juegan las modas. La psicología como ciencia no está exenta de generar focos de atención que se convierten en modas y dejan de lado o desdibujan otros ámbitos de investigación. En este sentido, cabe señalar que, en estos momentos, se observa un intento de englobar bajo el foco de la regulación emocional la totalidad de la conducta psicopatológica. Sin duda, las emociones y el estudio acerca de cómo regularlas son aspectos centrales que la ciencia cognitiva ha puesto felizmente en primer término. Ahora bien, esto no debería dejar de lado o minusvalorar la cognición de la que tanto hemos aprendido. Sensatamente habría que adoptar una perspectiva integral que permita analizar en profundidad la inexorable interacción que existe entre emoción, cognición y conducta, tanto desde una perspectiva intraindividual como interaccional.

2. Esto responde a una problemática que excede al terreno específico de la regulación emocional, ya que supone una manifestación de la visión psicopatológica que siempre ha prevalecido y que el nuevo RDoc parece mantener. 


\section{Conclusiones}

En las últimas dos décadas se ha producido un incremento exponencial de estudios en torno a la regulación emocional en los trastornos emocionales. Uno de los desafíos que impone el futuro inmediato consiste en priorizar aquellas líneas que puedan tener una significación clínica más notoria, de modo que el conocimiento pueda ser trasladado a la práctica en pos de reducir el sufrimiento de las personas que sufren trastornos emocionales. A este respecto, cabe recordar que esos trastornos son, precisamente, los más prevalentes en salud mental y, a la vez, aquellos que están menos atendidos. Además, no solo habría que prestar atención a los muchos pacientes que sufren y no reciben la atención necesaria, ya que existe también una importante cantidad de personas con trastornos emocionales subclínicos que también experimentan notable sufrimiento y tienen altas probabilidades de desarrollar cuadros más graves ante situaciones estresantes. Los abordajes transdiagnósticos que sustentan parte importante de su conceptualización en los procesos básicos de regulación emocional forman parte de estrategias que permitirán eliminar la brecha existente entre la ciencia y la práctica. En ese sentido, resulta sumamente relevante que se siga indagando en el terreno de la regulación emocional.

\section{Referencias}

Abramowitz, J. S., Tolin, D. F. y Street, G. P. (2001). Paradoxical effects of thought suppression: A meta-analysis of controlled studies. Clinical Psychology Review, 21, 683-703.

Aldao, A. (2012). Emotion regulation strategies as transdiagnostic processes: A closer look at the invariance of their form and function. Revista de Psicopatología y Psicología Clínica, 17, 261-277.

Aldao, A., Jazaieri, H., Goldin, P. R. y Gross, J. J. (2014). Adaptive and maladaptive emotion regulation strategies: interactive effects during CBT for social anxiety disorder. Journal of Anxiety Disorders, 28, 382-389.

Aldao, A., Nolen-Hoeksema, S. y Schweizer, S. (2010). Emotion-regulation strategies across psychopathology: A meta-analytic review. Clinical Psychology Review, 30, 217-237.

Aldao, A. y Tull, M. T. (2015). Putting Emotion Regulation in Context. Current Opinion in Psychology, 3, 100-107.

Beck, A. T. y Haigh, E. A. P. (2014). Advances in Cognitive Theory and Therapy: The Generic Cognitive Model*. Annual Review of Clinical Psychology, 10, 1-24.

Bryant, F. B. y Veroff, J. (2007) Savoring: A new model of positive experience. Mahwah, NJ, US: Lawrence Erlbaum Associates Publishers.

Bonanno, G. A., Papa, A., Lalande, K., Westphal, M. y Coifman, K. (2004). The Importance of Being Flexible. Psychological Science, 15, 482-487.

Boulanger, J. L., Hayes, S. C. y Pistorello, J. (2010). Experiential Avoidance as a Functional Contextual Concept. En A. M. Kring y D. M. Sloan (eds.), Emotion Regulation and Psychopathology: A Transdiagnostic Approach to Etiology and Treatment (pp. 107-136). New York: Guildford.

Brown, T. y Barlow, D. (2009). A proposal for a dimensional classification system based on the shared features of the DSM-IV anxiety and mood disorders: Implications for assessment. Psychological Assessment, 21, 256-271. 
Brown, T. A., Campbell, L. A., Lehman, C. L., Grisham, J. R. y Mancill, R. B. (2001). Current and lifetime comorbidity of the DSM-IV anxiety and mood disorders in a large clinical sample. Journal of Abnormal Psychology, 110, 585-599.

Campbell-Sills, L., Barlow, D. H., Brown, T. A. y Hofmann, S. G. (2006). Acceptability and suppression of negative emotion in anxiety and mood disorders. Emotion, 6, 587-595.

Campbell-Sills, L., Ellard, K. K. y Barlow, D. H. (2013). Emotion regulation in anxiety disorders. En J. J. Gross (ed.), Handbook of emotion regulation (2. ${ }^{a}$ ed.). New York: Guilford Press.

Carl, J. R., Soskin, D. P., Kerns, C. y Barlow, D. H. (2013). Positive emotion regulation in emotional disorders: A theoretical review. Clinical Psychology Review, 33, 343-360.

Eifert, G. H. y Heffner, M. (2003). The effects of acceptance versus control contexts on avoidance of panic-related symptoms. Journal of Behavior Therapy and Experimental Psychiatry, 34, 293-321.

Feldman, G. C., Joormann, J. y Johnson, S. L. (2008). Responses to positive affect: A selfreport measure of rumination and dampening. Cognitive Therapy and Research, 32, 507525

Gross, J. J. (1998). The emerging field of emotion regulation: An integrative review. Review of General Psychology, 2, 271-299.

Gross, J. J. (2015). Emotion regulation: Current status and future prospects. Psychological Inquiry, 26, 1-26.

Hayes, S. C., Strosahl, K. D. y Wilson, K. G. (1999). Acceptance and commitment therapy: An experiential approach to behavior change. New York: Guilford Press.

Hofmann, S. G. (2014). Interpersonal Emotion Regulation Model of Mood and Anxiety Disorders. Cognitive Therapy and Research, 38, 483-492.

Hofmann, S. G., Heering, S., Sawyer, A. T. y Asnaani, A. (2009). The effects of Reappraisal, Acceptance and Suppression Strategies on Anxious Arousal. Behavior Research \& Therapy, 47, 389-394.

Johnson, D. P. y Whisman, M. A. (2013). Gender differences in rumination: A meta-analysis, 55, 367-374.

Kring, A. M. y Sloan, D. S. (2010). Emotion regulation and psychopathology. New York, NY: Guilford Press.

Lilienfeld, S. y Treadway, M. T. (2016). Clashing Diagnostic Approaches: DSM-ICD versus RDoC. Annual Review of Clinical Psychology, 12, 435-465.

Nezlek, J. B. y Kuppens, P. (2008). Regulating positive and negative emotions in daily life. Journal of Personality, 76, 561-579.

Nolen-Hoeksema, S. (2012). Emotion Regulation and Psychopathology: The Role of Gender. Annual Review of Clinical Psychology, 8, 161-187.

Nolen-Hoeksema, S., Wisco, B. E. y Lyubomirsky, S. (2008). Rethinking Rumination. Perspectives on Psychological Science, 3, 400-424.

Papageorgiou, C. y Wells, A. (2001). Metacognitive beliefs about rumination in recurrent major depression. Cognitive and Behavioral Practice, 8, 160-164.

Purdon, C. (1999). Thought suppression and psychopathology. Behaviour Research and Therapy, 37, 1029-1054.

Scherer, A., Boecker, M., Pawelzik, M., Gauggel, S. y Forkmann, T. (2015). Emotion suppression, not reappraisal, predicts psychotherapy outcome. Psychotherapy Research. DOI: 10.1080/10503307.2015.1080875 
Sheppes, G., Suri, G. y Gross, J. J. (2015). Emotion Regulation and Psychopathology. Annual Review of Clinical Psychology, 11, 379-405.

Tamir, M. (2011). The maturing field of emotion regulation. Emotion Review, 3, 3-7.

Watkins, E. (2015). Psychological treatment of depressive rumination. Current Opinion in Psychology, 4, 32-36.

Wolfgast, M. (2012). Acceptance and Cognitive Restructuring. Analyses and comparisons from an emotion regulation perspective. Tesis doctoral. Universidad Lund, Malmö, Suecia. 